\title{
2D Materials Based Hybrid for Efficient Removal of Heavy Metal Ions
}

\author{
Agnieszka Mikołajczak ${ }^{1,2}$, Samanta Witomska ${ }^{1,2}$, Włodzimierz Czepa ${ }^{1,2}$, Artur Ciesielski ${ }^{2,3}$, Paolo Samorì ${ }^{3}$ \\ ${ }^{1}$ Faculty of Chemistry, Adam Mickiewicz University in Poznań \\ Umultowska 89b, 61-614 Poznań, Poland \\ ${ }^{2}$ Centre for Advanced Technologies, Adam Mickiewicz University \\ Umultowska 89c, 61-614 Poznań, Poland \\ agnmik4@st.amu.edu.pl; samanta.witomska@amu.edu.pl; wlodzimierz.czepa@amu.edu.pl \\ ${ }^{3}$ Nanochemistry Laboratory, ISIS \& icFRC, Université de Strasbourg \& CNRS \\ 8 allée Gaspard Monge, 67000 Strasbourg, France \\ ciesielski@unistra.fr; samori@unistra.fr
}

\section{Extended Abstract}

Due to fast growing industry, development of infrastructure and agriculture, more and more anthropogenic pollutants are released into the hydrosphere every year. Because of that, there is a huge demand for effective and low-cost methods for heavy metal ions removal from water. There are several different methods to dispose heavy metals from water including ion exchange, coagulation, biodegradation and adsorption. Among these methods the most well-known and commonly used is adsorption, mostly because of simple and easy operation, high efficiency and low cost. [1] Two-dimensional materials like graphene, hexagonal boron nitride and MXenes have high surface-to-volume ratio and two planar surfaces available for adsorption. [2] Considering hydrophilic nature of graphene, graphene oxide (GO) shows better potential in the removal of ions from aqueous solutions, with its strong hydrophilic nature and large surface area. [3] Moreover GO, as a highly oxidized form of graphene, contains various functional groups including hydroxyl, carboxyl and epoxide units on the surface. These groups can act as a reactive side for further functionalization. By covalently attaching groups with high affinity towards specific metals to GO, it is possible to create an effective adsorbent. So far, many GO-based hybrids have shown promising adsorbents applications. [4] Noteworthy, through thiol-carboxylic acid esterification reaction sulfur compounds can be covalently bonded onto GO surface and there are able to form stable complexes with soft heavy metals of high polarizability like $\mathrm{Hg}, \mathrm{Au}, \mathrm{Ag}$.

Firstly, the thiourea-formaldehyde polymer (TF) was prepared by hydroxyl-methylation and condensation reaction between thiourea and formaldehyde. The material was characterized using ${ }^{1} \mathrm{HNMR}$ spectroscopy, Fourier transform infrared spectroscopy (FT-IR) and elemental analysis. The weight-average molecular weights $\left(\mathrm{M}_{\mathrm{w}}\right)$ of TF polymer, as determined by diffusion-ordered NMR spectroscopy (DOSY NMR), amounted to $969 \mathrm{~g} / \mathrm{mol}$. The functionalization of GO was carried out by heating GO aqueous solution with TF in DMF for $24 \mathrm{~h}$. The unreacted polymer molecules were washed away, and the obtained material was freeze-dried for $24 \mathrm{~h}$ to sustain the high porosity and specific surface area. The resulting black solid was characterized using X-ray photoelectron spectroscopy (XPS), Fourier transform infrared spectroscopy (FT-IR), scanning electron microscope (SEM), energy-dispersive X-ray spectroscopy (EDX) analysis. Adsorption experiments were carried out with 3 or $5 \mathrm{mg}$ of adsorbents and 15 and $30 \mathrm{ml}$ of $\mathrm{Hg}^{2+}$ and $\mathrm{Au}^{3+}$ aqueous solutions, respectively. The solutions had desired concentration from 5 to $500 \mathrm{mg} / \mathrm{L}$ and appropriate $\mathrm{pH}$. The experiment last $24 \mathrm{~h}$ and then the concentration of remaining ions was measured.

This porous hybrid material based on TF polymer (G3DTF) is particularly appealing as an adsorbent of heavy metal ions. Through the homogeneous porous structure and BET surface area $402.51 \mathrm{~m}^{2} / \mathrm{g}$ of G3DTF, high maximum adsorption capacities towards heavy metal ions at $\mathrm{pH} 1$ were obtained. The maximum adsorption capacity for $\mathrm{Hg}^{2+}$ and $\mathrm{Au}^{3+}$ correlated with Langmuir isotherm were around $1460 \mathrm{mg} / \mathrm{g}$ and $2360 \mathrm{mg} / \mathrm{g}$, respectively. Further experiments are planned, but the presented results demonstrate that this new hybrid material could be considered as an alternative adsorbent for removal of ions from aqueous solutions. 


\section{References}

[1] D. Xu, J. Lu, S. Yan and R. Xiao, "Aminated EVOH nanofiber membranes for Cr(vi) adsorption from aqueous solution," $R S C A d v$. , vol. 8, no. 2, pp. 742-751, 2018.

[2] D. Pakulski, W. Czepa, S. Witomska, A. Aliprandi, P. Pawluć, V. Patroniak, A. Ciesielski and P. Samori, "Graphene oxide-branched polyethylenimine foams for efficient removal of toxic cations from water," J. Mater. Chem. A, vol. 6, no. 20, pp. 9384-9390, 2018.

[3] A. S. Krishna Kumar, S.-J. Jiang, W.-L. Tseng "Facile synthesis and characterization of thiol-functionalized graphene oxide as effective adsorbent for Hg(II)," J. Environ. Chem. Eng., vol. 4, no. 2, pp. 2052-2065, 2016.

[4] Z. Wang, X. Li, H. Liang, J. Ning, Z. Zhou and G. Li, "Equilibrium, kinetics and mechanism of $\mathrm{Au}^{3+}, \mathrm{Pd}^{2+}$ and $\mathrm{Ag}^{+}$ions adsorption from aqueous solutions by graphene oxide functionalized persimmon tannin," Mater. Sci. Eng. C., vol. 79, pp. 227-236, 2017. 